\title{
Impact of overweight and obesity on postmenopausal breast cancer: analysis of 20-year data from Switzerland
}

\author{
Monika Eichholzer • Seraina Margaretha Schmid • \\ Florence Bovey • Paul Jordan · Sabine Rohrmann • \\ Dorothy Jane Huang • Christoph Rochlitz • Uwe Güth
}

Received: 3 February 2011/Accepted: 13 July 2011 / Published online: 4 August 2011

(c) Springer-Verlag 2011

\begin{abstract}
Purpose Greater body fatness has been identified as a risk factor for postmenopausal breast cancer. For countries with low overweight/obesity rates, data on prevalence and time course of overweight/obesity in women with breast cancer in comparison to women in the general population is limited. The Swiss female population is distinctive for two reasons: (a) low rates of overweight/obesity compared with other western countries, and (b) no obesity epidemic, i.e. stable rates of overweight/obesity for more than 10 years. Methods Overweight and obesity were analyzed in 51 to 80 -year-old breast cancer patients initially diagnosed between 1990 and 2009. Patient data was derived from the Basel Breast Cancer Database (BBCD). This data was compared with the data of women of the same age from the
\end{abstract}

M. Eichholzer · F. Bovey · P. Jordan · S. Rohrmann

Institute of Social and Preventive Medicine,

University of Zurich, Hirschengraben 84,

8001 Zurich, Switzerland

S. M. Schmid · D. J. Huang · U. Güth

Department of Gynecology and Obstetrics,

University Hospital Basel (UHB), Spitalstrasse 21,

4031 Basel, Switzerland

S. M. Schmid · C. Rochlitz · U. Güth

Breast Centre, University Hospital Basel (UHB),

Spitalstrasse 21, 4031 Basel, Switzerland

C. Rochlitz

Department of Medical Oncology, University Hospital Basel

(UHB), Petersgraben 4, 4031 Basel, Switzerland

U. Güth $(\square)$

Department of Gynecology and Obstetrics, Cantonal Hospital

Winterthur, Brauerstrasse 15, 8401 Winterthur, Switzerland

e-mail: uwe.gueth@unibas.ch four Swiss Health Surveys (SHS) conducted between 1992 and 2007. Differences between measured (BBCD) and selfreported (SHS) data were corrected using equations approved for the Swiss population.

Results Of 958 postmenopausal BBCD patients, 32\% were overweight and $20 \%$ were obese. Of the 14,476 women of the SHS, $38 \%$ were overweight and $17 \%$ were obese. In the BBCD, there was no change in the prevalence of overweight/obesity over the last 20 years. The four SHS show a convex curvature for obesity, i.e. a transient increase. No significant differences were observed between BBCD and corrected SHS data for overweight and obesity during this period.

Conclusions In this Swiss study group with a comparably low prevalence of overweight and obesity, no association between body fatness and postmenopausal breast cancer was observed.

Keywords Breast cancer - Overweight - Obesity · Risk factor · Epidemiology - Swiss Health Survey

\section{Introduction}

In 2007, based on an extensive literature review, the working group World Cancer Research Fund (WCRF)/ American Institute for Cancer Research (AICR) came to the conclusion that there is consistent epidemiological evidence and a clear dose response regarding greater body fatness as a risk factor for postmenopausal breast cancer [1].

The present study analyses the relationship between overweight/obesity and breast cancer risk using Swiss data of a 20-year period. With regard to the prevalence of obesity, the Swiss female general population is, according 
to the Swiss Health Surveys [2-4], distinctive in two areas compared to most other industrialized countries [5, 6]: Switzerland has comparatively low rates of uncorrected self-reported overweight (22-23\%) and obesity (7-8\%) of women aged 18 years and older and has no obesity epidemic, i.e. remarkably stable rates of overweight and obesity over the past 10 years.

As the dose-response relationship between BMI and postmenopausal breast cancer might be curvilinear (exponential), an increased risk may only be demonstrated in a population with a high prevalence of obese and very obese women. Thus it is of interest whether the consistently shown association between obesity and breast cancer risk in countries with high obesity prevalence rates [1] can also be shown in a population with a rather low obesity prevalence.

\section{Patients and methods}

\section{Datasource 1: Basel Breast Cancer Database}

The Basel Breast Cancer Database (BBCD) comprises all newly diagnosed primary invasive breast cancer cases treated at the University Women's Hospital Basel, Switzerland, since 1990. For this study, data from patients up to and including 2009 were analyzed $(n=1,495)$. All data were recorded retrospectively from the medical files. Recording and storage of the data was done in a web-based documentation system. Data collection methods and study design were approved by the Ethical Review.

\section{Datasource 2: Swiss Health Survey}

The Swiss Health Survey (SHS) has been conducted on behalf of the Federal Office of Statistics since 1992 in fiveyear intervals. The already existing four surveys, from 1992, 1997, 2002 and in 2007, were each carried out in representative, randomly selected samples comprising 20,000 to 30,000 private households with a telephone connection to represent the Swiss permanent population, i.e. male and female Swiss citizens and foreigners with a legal work permit aged 15 years and older [7].

Study cohort

In this study, it was our aim to evaluate postmenopausal women. Although we have close to complete information regarding the menopausal status of the patients in the $\mathrm{BBCD}$, we do not have this information in the data from the SHS. Since the mean age of menopause in the BBCD was 50.06 years, we used an age-dependent inclusion criterion and analyzed women from 51-80 years of age.
Data on Body Mass Index in BBCD and SHS

In the $\mathrm{BBCD}$, measured weight and height were retrieved retrospectively from medical files. In the SHS, respondents were asked in a telephone interview to report their height (without shoes) and weight (without clothes).

Based on these data, body mass index (BMI = body weight $\left[\mathrm{kg} / \mathrm{height} \mathrm{m}^{2}\right]$ ) was calculated and categorized according to the WHO criteria as follows [8]:

- BMI <18.5: underweight

- BMI 18.5-24.9: normal (desirable) weight

- BMI 25-29.9: overweight

- $\quad$ BMI $\geq 30$ : obesity

In the BBCD, body weight and height have been collected continuously since 1990. However, in the SHS, this data was collected solely in the four years that the surveys were performed, namely 1992, 1997, 2002 and 2007. In order to evaluate BMI distribution time trends over the last 20 years, we defined the four following subgroups:

- BBCD 1990-1994 versus SHS 1992

- BBCD 1995-1999 versus SHS 1997

- BBCD 2000-2004 versus SHS 2002

- BBCD 2005-2009 versus SHS 2007

In this study, we compared objective BMI data (BBCD: measured) with subjective ones (SHS: self-reported). Since individuals included in population studies tend to underreport their weight and overestimate their height, obesity prevalence based on this data is often inaccurate [9]. Since over- and underreporting appears to be quite systematic, correction factors using separate adjustment factors could increase the accuracy of self-reported estimates [10-12]. In this study, we used an equation developed by Hayes et al. [11]. This calculation model was tested and approved for the Swiss population [13].

\section{Statistical methodology}

To allow for non-responders and stratification in relation to age, gender, and nationality in the four SHS, differences between the samples and the permanent Swiss population were mathematically corrected and weighted respectively with the corresponding formula provided by the Swiss Federal Office of Statistics [7].

In order to evaluate the time courses for the different BMI categories, cases were dichotomized with respect to the corresponding class. As an example: A person belonging to the category "overweight" was assigned to the outcome " 1 " for the outcome variable "Overweight Yes/No" and to the value " 0 " for the other binary outcome 
variables "Underweight Yes/No", "Normal Weight Yes/ No" and "Obesity Yes/No".

For each of the binary outcome variables, for both data sources (BBCD and SHS) the following logistic regression model was applied:

$\operatorname{logit}\left(\mathrm{Z}_{i j}\right)=\mu+\alpha_{j(*)}$

where the terms in this model are $Z_{i j}$ is the Binary outcome for subject $\mathrm{i}$ at year $\mathrm{j}$. 1: if the person belongs to the corresponding BMI category; 0 else, and $\mu$ the grand mean, and $\alpha_{j}$ the effect of year $\mathrm{j}$ (factor variable with 4 levels: 1992, 1997, 2002, 2007)

If the overall effect of 'year' was significant at the 5\%level, the linear and quadratic contrasts were tested. The first one describes a linear (decreasing or increasing) trend, the second one a possible convex or concave ("U = shaped") time course. For the SHS studies, the data was weighted to allow for non-responders and stratification in relation to age, gender, and nationality. The confidence limits for the weighted frequencies in the SHS studies were also calculated by means of a logistic model as (*) with "initial weights".

Confidence intervals and tests with unweighted data are slightly different from the weighted ones presented here; the interpretation of the results, however, remains the same.

All analyses were conducted using SAS, version 9.1 for data handling and $\mathrm{R}$, version 2.9 , for the calculation of the statistical models and for the graphical representations.

\section{Results}

Of 1,495 patients in the BBCD, 985 (65.7\%) were 51-80 years old at diagnosis. Of these, information on BMI was available for 958 women and, thus, was analyzed in the study; the mean age of this study subgroup was 65.0 years. From the 35,090 women who were interviewed in the SHS and provided information on BMI, 14,476 women $(37.2 \%)$ met our age-dependent inclusion criteria; the mean, weighted age of this subgroup was 63.7 years.

BMI distribution in women of the BBCD and the SHS

BMI distributions of the BBCD and the SHS study cohorts are shown in Table 1. Of the BBCD group, 309 women $(32.3 \%)$ were overweight (BMI 25-29.9) and 188 women $(19.6 \%)$ were obese $(\mathrm{BMI} 30+)$ at the time of the initial breast cancer diagnosis. In comparison, of women of the same age in the overall group of the SHS 1992-2007 ( $n=14,476), 37.7 \%$ were overweight and $17.0 \%$ were obese (corrected for self-report [11, 13]).

How did the BMI distribution develop over the last 20 years?

During the four time periods considered in the BBCD (1990-94, 1995-99, 2000-04, 2005-09), the prevalence of overweight were $35.5,36.3,31.3$ and $27.8 \%$, respectively; the prevalence of obesity were $15.0,23.8,18.9$, and $21.2 \%$, respectively. (Table 2, Fig. 1). For 51 to 80-year-old women of the four SHS of 1992, 1997, 2002 and 2007, the corresponding prevalence for overweight and obesity, corrected for self-report $[11,13]$, were 38.6, 37.8, 38.5, 36.2 and $12.9,19.5,17.4$ and $17.7 \%$, respectively.

Statistical tests for the overall time course over the four periods revealed a comparable pattern in all tested categories of the SHS and the BBCD, respectively (Table 2). For "overweight", the tests for the overall time course were not statistically significant. For "obesity", the test for the overall time course was highly significant in the SHS; the corresponding linear contrast was positive and statistically significant, indicating an increase over time. Additionally, the quadratic contrast was significant and

Table 1 BMI distribution in 51-80 years old women of the Basel Breast Cancer Database (BBCD) between 1990 and 2009 in comparison to women of the same age in the Swiss Health Surveys (SHS) 1992-2007

\begin{tabular}{|c|c|c|c|c|}
\hline \multirow[t]{2}{*}{ Body Mass Index } & \multicolumn{2}{|c|}{ BBCD 1990-2009 } & \multicolumn{2}{|c|}{ SHS $1992-2007^{\mathrm{a}}$} \\
\hline & $N$ & $\%$ & $N^{\mathrm{b}}$ & $\%$ \\
\hline Underweight BMI $<18.5 \mathrm{~kg} / \mathrm{m}^{2}$ & 22 & 2.3 & 203 & 1.4 \\
\hline Normal weight BMI $18.5-24.9 \mathrm{~kg} / \mathrm{m}^{2}$ & 439 & 45.8 & 6,355 & 43.9 \\
\hline Overweight BMI $25-29.9 \mathrm{~kg} / \mathrm{m}^{2}$ & 309 & 32.3 & 5,457 & 37.7 \\
\hline Obesity BMI $30+\mathrm{kg} / \mathrm{m}^{2}$ & 188 & 19.6 & 2,461 & 17.0 \\
\hline
\end{tabular}


Table 2 Statistical tests to evaluate the overall time course of overweight and obesity prevalence in 51 to 80 -year-old women of the BBCD from 1990 to 2009 in comparison to women of the same age in the SHS 1992, 1997, 2002 and 2007

\begin{tabular}{|c|c|c|c|c|}
\hline \multirow[t]{2}{*}{ Study group } & \multirow[t]{2}{*}{ BMI category } & \multicolumn{3}{|l|}{ 1990-2007 } \\
\hline & & $\begin{array}{l}\text { "year" (overall } \\
\text { time course) }\end{array}$ & Linear trend & Quadratic trend \\
\hline \multirow[t]{2}{*}{$\mathrm{BBCD}^{\mathrm{a}, \mathrm{b}}$} & Overweight & ns & & \\
\hline & Obesity & $\mathrm{ns}$ & & \\
\hline \multirow[t]{2}{*}{$\mathrm{SHS}^{\mathrm{c}, \mathrm{d}, \mathrm{e}, \mathrm{f}}$} & Overweight & $\mathrm{ns}$ & & \\
\hline & Obesity & $* * *$ & $+; * * *$ & $-; * * *$ \\
\hline
\end{tabular}

$n s$ statistically not significant, $* * * p<0.001,+$ a positive linear trend or a concave quadratic trend, - a negative linear trend or a convex quadratic trend

a $B B C D$ Basel Breast Cancer Database

b Compared time periods: 1990-1994, 1995-1999, 2000-2004, 2005-2009

c SHS Swiss Health Survey, Datasource: Swiss Federal Office (FSO)

${ }^{\mathrm{d}}$ Weighted to allow for non-responders and stratification in relation to age, gender, and nationality

e Compared time periods 1992, 1997, 2002, 2007

${ }^{\mathrm{f}}$ Data corrected for self-report [13]

Fig. 1 BMI distribution in 51-80 years old women of the Basel Breast Cancer Database (BBCD) between 1990 and 2009 in comparison to women of the same age in the Swiss Health Surveys (SHS, corrected for self-report [13]). Datasource of the SHS: Swiss Federal Office (FSO); 1997, underweight $n=28$ weighted, with $95 \%$ CI 1992, 1997, 2002, and 2007
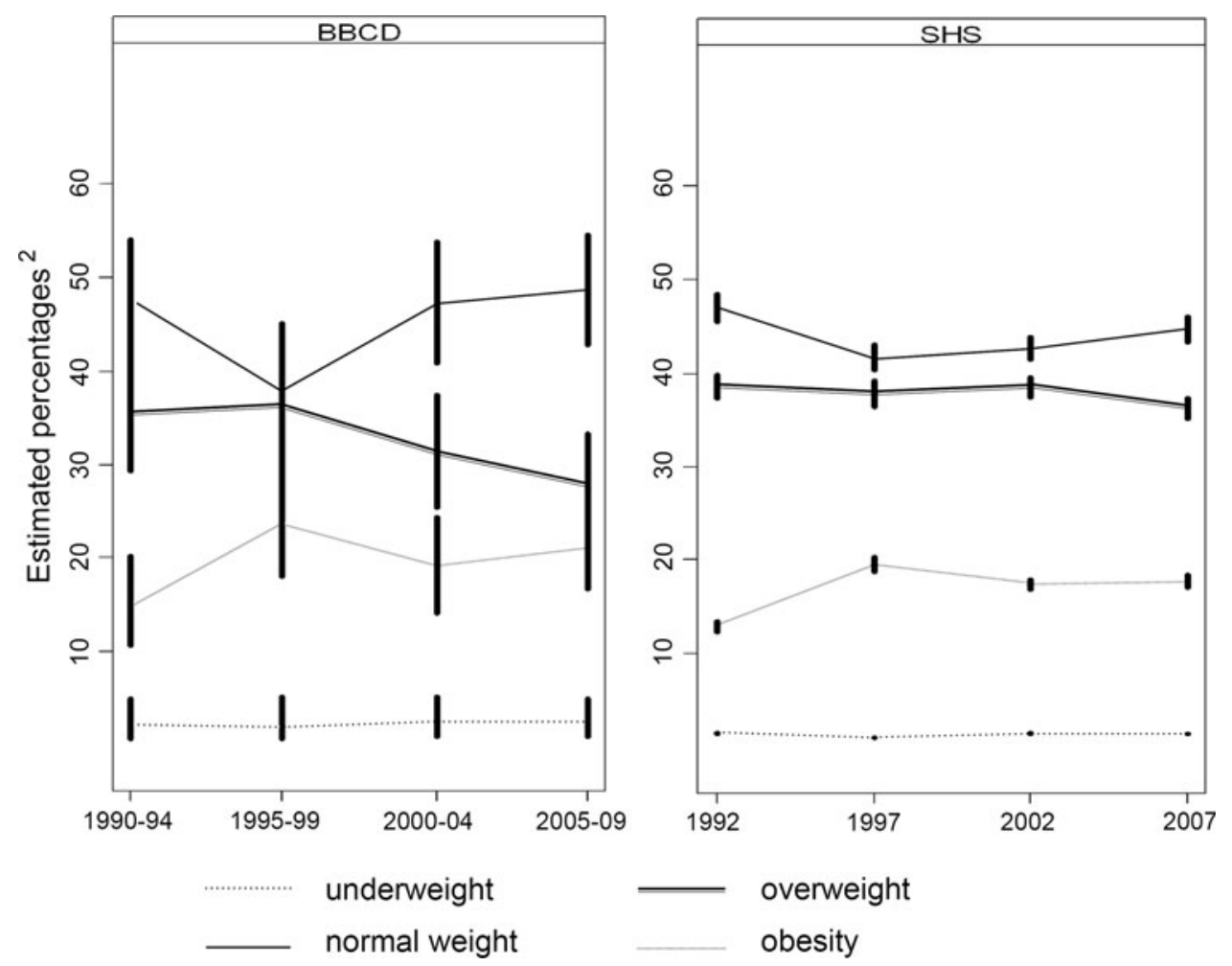

negative, indicating a convex curvature, as can be seen in Fig. 1. For the BBCD data, however, the test for the overall time course was not statistically significant.

Was there a higher prevalence of overweight and obesity in patients with breast cancer than in women of the general population?

Overall, there was no evidence of statistically significant differences between BBCD data and corrected and weighted SHS data in relation to overweight and obesity over the four time periods considered (Table 3).

\section{Discussion}

Body fatness increases blood levels of a number of hormones, such as estrogens, insulin-like growth factors and insulin, thereby creating an environment that facilitates carcinogenesis [14]. Multiple epidemiological lines of evidence have shown that overweight and obesity pose a risk factor for postmenopausal breast cancer. In a metaanalysis conducted in 2007 by the WCRF/AICR on the basis of 17 cohort and 48 case-control studies, the authors 
Table 3 Overweight (BMI 25-29.9) and obesity (BMI 30+) in 51-80 years old postmenopausal women of the BBCD from 1990 to 2009 in comparison to women of the same age in the SHS 1992, 1997, 2002 and 2007

\begin{tabular}{|c|c|c|c|c|c|}
\hline \multirow[t]{2}{*}{ Time period } & \multirow[t]{2}{*}{ Study group } & \multicolumn{2}{|c|}{ Overweight } & \multicolumn{2}{|c|}{ Obesity } \\
\hline & & $\%$ & $95 \% \mathrm{CI}$ & $\%$ & $95 \%$ CI \\
\hline 1990-1994 & $\mathrm{BBCD}^{\mathrm{a}}$ & 35.5 & $29.4-42.0$ & 15.0 & $10.6-20.2$ \\
\hline 1992 & $\mathrm{SHS}^{\mathrm{b}, \mathrm{c}, \mathrm{d}}$ & 38.6 & $37.3-39.8$ & 12.9 & $12.4-13.5$ \\
\hline 1995-1999 & $\mathrm{BBCD}^{\mathrm{a}}$ & 36.3 & $29.5-43.5$ & 23.8 & $18.0-30.5$ \\
\hline 1997 & $\mathrm{SHS}^{\mathrm{b}, \mathrm{c}, \mathrm{d}}$ & 37.8 & $36.5-39.1$ & 19.5 & $18.8-20.3$ \\
\hline 2000-2004 & $\mathrm{BBCD}^{\mathrm{a}}$ & 31.3 & $25.5-37.5$ & 18.9 & $14.2-24.4$ \\
\hline 2002 & $\mathrm{SHS}^{\mathrm{b}, \mathrm{c}, \mathrm{d}}$ & 38.5 & $37.4-39.6$ & 17.4 & $16.8-18.0$ \\
\hline 2005-2009 & $\mathrm{BBCD}^{\mathrm{a}}$ & 27.8 & $22.7-33.3$ & 21.2 & $16.6-26.4$ \\
\hline 2007 & $\mathrm{SHS}^{\mathrm{b}, \mathrm{c}, \mathrm{d}}$ & 36.2 & $35.2-37.3$ & 17.7 & $17.1-18.3$ \\
\hline
\end{tabular}

${ }^{\text {a }} B B C D$ Basel Breast Cancer Database

b SHS Swiss Health Survey; Datasource: Swiss Federal Office (FSO)

c Weighted to allow for non-responders and stratification in relation to age, gender, and nationality

d Data corrected for self-report [13]

calculated a summary effect estimate of $1.03(95 \% \mathrm{CI}$ $1.01-1.04)$ and $1.05(95 \%$ CI $1.05-1.06)$ per $2 \mathrm{~kg} / \mathrm{m}^{2}$ increase in BMI, respectively [1]. Therefore, one would expect that, relating to a defined population of postmenopausal women, there are more overweight and obese women in a breast cancer cohort than in the general population. In the present analysis no such difference was observed for the Swiss study group. Out of the postmenopausal breast cancer patients of the BBCD, 32\% were overweight and $20 \%$ were obese. This data is comparable with a group of 337 breast cancer patients $>50$ years who were registered in the canton of Geneva between 2003 to 2005 and of which $28 \%$ were overweight and $21 \%$ were obese [15]. In comparison, of the 51 to 80 years old women of the overall group of the SHS 1992 to 2007, 38\% were overweight and 17\% were obese (corrected for self-report $[11,13])$. In the BBCD, the overweight/obesity rates have been more or less consistent over the last 20 years. In the course of the four SHS, a convex pattern, i.e. transient increase in the prevalence of obesity was observed. For the whole group of women in the SHS, i.e. including women 18 years and older, overweight and obesity increased between 1992 and 1997, but between 1997 and 2007, however, no statistically significant increase was observed anymore [4]. Likewise, in a cross-sectional repeated survey of adults aged 35-74 years in Geneva, obesity prevalence did not increase significantly in women between 1993 and 2004 [16]. This data contradicts that of most other industrialized countries which have been affected by a considerable rise in obesity prevalence ("global epidemic") [17-22]. Compared to the USA, the obesity rates in Switzerland are actually half as high [6].

A possible explanation for the fact that our data is not in alignment with most other studies analyzing the relationship between body fatness and postmenopausal breast cancer could be due to the lower prevalence of female overweight and obesity in comparison to other European countries [5, 22]. In the four Swiss Health Surveys in women aged 18 years and older rates of uncorrected self-reported overweight were between 22 and $23 \%$ and of obesity between 7 and $8 \%$ [4]. Data of the International Association for the Study of Obesity (IASO) on self-reported weight and height, collected in women between 1997 and 2008 in the European Union, revealed prevalence of overweight between $24 \%$ in Belgium and $34 \%$ in Malta and of obesity between $9 \%$ in Italy and $19 \%$ in Slovakia. The surveys used in this overview were not strictly comparable because of differences in age range and methodology [5]. However, the Swiss population is, in general as well as in different analyses taking into account gender- and age-specific subgroups, clearly at the lower end of this list.

As already mentioned, measured BMI data of the BBCD and BMI data of the SHS corrected for self-report revealed for women aged 51-80 years overweight prevalence of 32 and $38 \%$ and of obesity of 20 and $17 \%$. These quite high levels of overweight and obesity in comparison to the ones of women aged 18 years and more of the SHS most probably reflect the rather high average age of this group and the well-known increase of overweight prevalence with increasing age [4].

Findings from countries with high overweight/obesity prevalence (approximately $50 \%$ of the cohort studies compiled in the WCRF/AICR review stem from the USA [1]) may not unconditionally be applied to populations with comparably rather low overweight/obesity prevalence such as Switzerland. For example, in the USA there are significantly more women with excessive body fatness, a 
condition which may indicate a considerably different lifestyle, including numerous other factors that might also facilitate carcinogenesis. However, these factors can hardly be controlled for. A limitation of the present study is that no control for potential confounders other than age was possible.

Another possible explanation for the fact that in our study no association between overweight and obesity and postmenopausal breast cancer could be demonstrated may be the possibility of a curvilinear dose-response relationship between BMI and postmenopausal breast cancer risk [1], such that an increased risk may only be observed in population with a high prevalence of obese and very obese women.

Furthermore, body measurements that describe central obesity such as waist circumference and the waist-to-hip ratio might reflect the increased hazards of fat tissue related to increased breast cancer risk (i.e. to increase the bioactivity of circulating estrogens) better than the BMI alone [23].

In addition, overweight might be associated with an increased risk of postmenopausal breast cancer, especially among those who have never used hormone replacement therapy (HRT) [24]. In the BBCD, restriction of the analyses on women without HRT (64\%) (with HRT: 19\%, unknown: $17 \%$ ) was only possible for the overall group of breast cancer patients and revealed that $34 \%$ of women without HRT were overweight and $20 \%$ were obese; detailed analyses on time trends etc. were impossible due to the limited number of breast cancer cases.

Furthermore, our study, as most of the other epidemiological studies conducted so far, could not discriminate between the hormonal receptor positive and negative breast cancer.

Finally, unintentional weight loss related to their breast cancer in women of the BBCS can be excluded as an explanation of our negative results, because very few patients presented cancer-related cachexia.

Two other critical points must be considered in the interpretation of our data. Firstly, it is important to note that in the BBCD, women's weight and height were measured directly. In the SHS, on the contrary, this data was collected in a telephone interview, i.e. was self-reported. It is a well-known fact that self-reported body height is often overestimated and weight is underestimated and that therefore the BMI is underestimated [9, 25-27]. Worldwide, studies have been carried out to estimate the gap between self-reported and measured BMI data and correction equations have been proposed, e.g. [10-12]. These equations consider several population-specific selective criteria [27]. Using such an equation, it must be assured that it is applicable for the particular population analyzed. Comparing different methods of adjustment for the Swiss population, Fäh et al. [13] came to the conclusion, that equation adjustment had advantages over the use of an universal lower cut-off level. The authors tested several calculation models for correcting self-reported weight and height and found that by using correction equations from Hayes et al. [11] the self-reported data corresponded best to the measured ones in Switzerland [13]. As this equation was defined to represent Swiss characteristics, we used it in our study.

Secondly, many breast cancer studies do not use agedependent subgroups but differentiate between the menopausal status (pre- or postmenopausal). Our aim was to evaluate the group of postmenopausal women. Although we possessed almost complete information regarding the menopausal status of the patients in the BBCD, we did not have this information in the data from the SHS. Since the mean age of menopause in the BBCD was 50.06 years, we used the above-mentioned subdivision of age in our study.

We are aware of the fact that using this method would lead certain patients being included ( $>51$ years, premenopausal) or excluded ( $<51$ years, postmenopausal) in a debatable way. However, with respect to the entire study group $(n=958)$, the number of such patients is so small, that they should not have a significant effect on the reported results. Furthermore, a certain variance in time will hardly lead to the fact that the women will be classified into another BMI classification subgroup, which was our target variable in this study. In general, there is an increasing awareness both by clinicians and epidemiologists of the fact that in the evaluation of cohorts which are defined and differentiated by the menopausal status, a grey area in the transition between pre- and postmenopause is unavoidable, since a certain number of women can barely be fitted into a theoretical framework (e.g., the menopausal status is unclear due to the history of hysterectomy in the premenopausal situation or the application of hormonal measures) $[28,29]$.

In conclusion, we did not observe higher rates of overweight and obesity in breast cancer cases than in the general population. Thus, in our Swiss study group with comparably low prevalence rates of overweight and obesity, we could not show an association of overweight and obesity with postmenopausal breast cancer.

Acknowledgments The authors want to thank the Swiss Federal Office of Statistics for permission to use the data of the Swiss Health Survey 1992-2007. This study was supported by the "Krebsliga beider Basel", grant 09-2009. The study sponsor had no influence whatsoever on the reported data.

Conflict of interest The authors declare that there are no financial or personal relationships with other people or organizations that could inappropriately influence the work reported or the conclusions, implications, or opinions stated. 


\section{References}

1. AICR: World Cancer Research fund and American Institute for Cancer Research. Food, Nutrition, physical activity, and the prevention of cancer: A global perspective. Washington DC: AICR 2007, pp 218-221

2. Eichholzer M, Bernasconi F, Jordan P, Gutzwiller F (2005) Nutrition in Switzerland 2002-results of the Swiss Health Survey. Praxis (Bern 1994) 94(44):1713-1721

3. Eichholzer M, Bisig B, Gutzwiller F, Lüthy J (2000) Aktuelle Ernährungsprobleme in der Schweiz. Resultate der Schweizerischen Gesundheitsbefragung 1997. Mitt Lebensm Hyg 91:251-273

4. Eichholzer M, Bovey F, Jordan P, Probst-Hensch N, Stoffel-Kurt N (2010) Data on overweight and nutrition in the 2007 Swiss Health Survey. Praxis (Bern 1994) 99(1):17-25

5. International Association of Obesity (IASO). Overweight and Obesity in the EU27. Available at: http://www.iotf.org/database/ documents/v2PDFforwebsiteEU27.pdf

6. Flegal KM, Carroll MD, Ogden CL, Curtin LR (2010) Prevalence and trends in obesity among US adults, 1999-2008. JAMA; 303(3):235-241

7. Die Bundesbehörden der Schweizerischen Eidgenossenschaft. Erhebungen, Quellen-Schweizerische Gesundheitsbefragung (SGB). Available at: http://www.bfs.admin.ch/bfs/portal/de/ index/infothek/erhebungen_quellen/blank/blank/ess/01.html

8. World Health Organization (2003) Diet, nutrition and the prevention of chronic diseases. WHO-Technical Report Series no. 916. World Health Organization, Geneva

9. Gorber SC, Tremblay M, Moher D, Gorber B (2007) A comparison of direct vs. self-report measures for assessing height, weight and body mass index: a systematic review. Obes Rev 8(4):307-326

10. Connor Gorber S, Shields M, Tremblay MS, McDowell I (2008) The feasibility of establishing correction factors to adjust selfreported estimates of obesity. Health Rep 19(3):71-82

11. Hayes AJ, Kortt MA, Clarke PM, Brandrup JD (2008) Estimating equations to correct self-reported height and weight: implications for prevalence of overweight and obesity in Australia. Aust N Z J Public Health 32(6):542-545

12. Visscher TL, Viet AL, Kroesbergen IH, Seidell JC (2006) Underreporting of BMI in adults and its effect on obesity prevalence estimations in the period 1998 to 2001. Obesity (Silver Spring) 14(11):2054-2063

13. Faeh D, Braun J, Bopp M (2009) Underestimation of obesity prevalence in Switzerland: comparison of two methods for correction of self-report. Swiss Med Wkly 139(51-52):752-756

14. Manjer J, Kaaks R, Riboli E, Berglund G (2001) Risk of breast cancer in relation to anthropometry, blood pressure, blood lipids and glucose metabolism: a prospective study within the Malmo Preventive Project. Eur J Cancer Prev 10(1):33-42
15. Deglise C, Bouchardy C, Burri M et al (2009) Impact of obesity on diagnosis and treatment of breast cancer. Breast Cancer Res Treat 120(1):185-193

16. Wolff H, Delhumeau C, Beer-Borst S, Golay A, Costanza MC, Morabia A (2006) Converging prevalences of obesity across educational groups in Switzerland. Obesity (Silver Spring) 14(11):2080-2088

17. Charafeddine R, Van Oyen H, Demarest S (2009) Trends in social inequalities in obesity: Belgium, 1997 to 2004. Prev Med 48(1):54-58

18. Rennie KL, Jebb SA (2005) Prevalence of obesity in Great Britain. Obes Rev 6(1):11-12

19. Czernichow S, Vergnaud AC, Maillard-Teyssier L et al (2009) Trends in the prevalence of obesity in employed adults in centralwestern France: a population-based study, 1995-2005. Prev Med 48(3):262-266

20. Neovius M, Janson A, Rossner S (2006) Prevalence of obesity in Sweden. Obes Rev 7(1):1-3

21. Schokker DF, Visscher TL, Nooyens AC, van Baak MA, Seidell JC (2007) Prevalence of overweight and obesity in the Netherlands. Obes Rev 8(2):101-108

22. Andreyeva T, Michaud PC, van Soest A (2007) Obesity and health in Europeans aged 50 years and older. Public Health 121(7):497-509

23. Kaaks R, Van Noord PA, Den Tonkelaar I, Peeters PH, Riboli E (1998) DE: Breast-cancer incidence in relation to height, weight and body-fat distribution in the Dutch "DOM" cohort. Int J Cancer 76(5):647-651

24. Lahmann PH, Hoffmann K, Allen N et al (2004) Body size and breast cancer risk: findings from the European Prospective Investigation into Cancer And Nutrition (EPIC). Int J Cancer 111(5):762-771

25. Paccaud F, Wietlisbach V, Rickenbach M (2001) Body mass index: comparing mean values and prevalence rates from telephone and examination surveys. Rev Epidemiol Sante Publique 49(1):33-40

26. Faeh D, Marques-Vidal P, Chiolero A, Bopp M (2008) Obesity in Switzerland: do estimates depend on how body mass index has been assessed? Swiss Med Wkly 138(13-14):204-210

27. Dauphinot V, Wolff H, Naudin F et al (2009) New obesity body mass index threshold for self-reported data. J Epidemiol Community Health 63(2):128-132

28. Ortmann O, Pagani O, Jones A et al (2011) Which factors should be taken into account in perimenopausal women with early breast cancer who may become eligible for an aromatase inhibitor? Recommendations of an expert panel. Cancer Treat Rev 37(2):97-104

29. Rosner B, Colditz GA (2011) Age at Menopause: Imputing Age at Menopause for Women With a Hysterectomy With Application to Risk of Postmenopausal Breast Cancer. Ann Epidemiol Mar 25 [epub ahead of print] 